\title{
Constraints on the provenance of the uppermost allochthonous terrane of the NW Iberian Massif: inferences from detrital zircon $\mathrm{U}-\mathrm{Pb}$ ages
}

\author{
Javier Fernández-Suárez, ${ }^{1}$ Florentino Díaz García, ${ }^{2}$ Teresa E. Jeffries, ${ }^{3}$ Ricardo Arenas ${ }^{1}$ \\ and Jacobo Abati ${ }^{1}$ \\ ${ }^{1}$ Departamento de Petrología y Geoquímica, Universidad Complutense, $2844 \mathrm{Madrid}$, Spain; ${ }^{2}$ Departamento de Geología, Universidad de \\ Oviedo, 33005 Oviedo, Spain; ${ }^{3}$ Department of Mineralogy, The Natural History Museum, London SW7 5BD, UK
}

\begin{abstract}
A BSTRACT
Insight into the origin and pre-orogenic palaeogeographical links of terranes involved in the assembly of collisional mountain belts is fundamental to the understanding of orogenic processes. Here we address the provenance and possible tectonic settings of the uppermost allochthonous terrane of the NW Iberian Variscan Belt through a 213-nm Laser Ablation ICP-MS study of $\mathrm{U}-\mathrm{Pb}$ ages of detrital zircons. The age groups of zircons from greywackes in this terrane (c. 480-610, 1900-2100, 2400-2500 Ma) and the lack of Mesoproterozoic zircons suggest an origin in a Neoproterozoic - Early Palaeozoic peri-
\end{abstract}

Gondwanan realm along the periphery of the west African craton. It is further inferred that the greywackes were deposited in the periphery of a crustal unit that had been detached from the Gondwanan margin in relation to the opening of the Rheic ocean in Cambro-Ordovician times. This terrane was thrusted back upon the Gondwanan marg in during the course of the Variscan collision and closure of the intervening ocean.

\section{Introduction}

Present-day understanding of the Variscan Laurentia-Gondwana collision in Europe owes much to structural, metamorphic and geochronological studies carried out on the Alloch thonous Complexes (AC) of NW Iberia (e.g. Martinez Catalán et al., 1997, 1999, and references therein). These studies have provided first-order constraints on the tectonothermal events involved in the Laurentia-Gondwana collision within the western European realm of the Variscan belt.

A major issue that has not been fully addressed in previous studies is the provenance of the allochthonous terranes thrusted upon the Gondwanan margin (relative autochthon) during the course of the Variscan collision (e.g. Martinez Catalán et al., 1996, 1997, 1999; Matte, 2001). It is generally agreed that the autoch thonous terranes of NW Iberia (Fig. 1) were part of the Gondwanan continental margin, but there is little evidence as regards the parentage of the allochthonous terranes. This issue is directly connected to the controversy regarding the pre-Variscan evolution of these geological units

C•rrespøndence: Dr Javier FernándezSuárez, Departament• de Petrøløgía y Geøquímica, Universidad Complutense, 28040 Madrid, Spain. E-mail: jfsuarez@ ge•.ucm.es
(Martínez Catalán et al., 1997; Abati et al., 1999; Matte, 2001).

In this work we investigate the provenance and pre-Variscan tectonic settings of the uppermost terrane of the AC of NW Iberia through a study of detrital zircon $\mathrm{U}-\mathrm{Pb}$ ages in low-grade greywackes located in the uppermost structural unit of the nappe pile (Fig. 1). This study is the first of its kind carried out on the Variscan AC of Iberia and is aimed at providing new constrains on their tectonic and palaeogeographical evolution.

\section{Geological setting}

The Allochthonous Complexes of NW Iberia (Fig. 1), whose main geological features have been described in detail elsewhere (Martínez Catalán et al., 1999, and references therein), consist of units stacked upon a relative autochthon considered to be the continental margin of Gondwana. These complexes, exposed as structural synforms, are formed by units with different tectonothermal histories and are interpreted to be dismembered fragments of a Variscan accretionary complex (Martínez Catalán et al., 1997, 1999). Three tectonometamorphic units have been distinguished in the AC (Arenas et al., 1995) based on their structural position and tectonometamorphic evolution: basal, intermediate (ophiolitic) and upper units.
The basal units consist of schists, paragneisses, and Early Ordovician felsic and mafic igneous rocks that underwent subduction and high-pressure metamorphism at $38-365 \mathrm{Ma}$ during the closure of an oceanic realm (Arenas et al., 1995; Martinez Catalán et al., 1996). They are considered to represent the external edge of the Gondwanan margin involved in the collision. The ophiolitic unit (Díaz García et al., 1999) structurally overlies the basal units and represents an Early Devonian oceanic crust. The upper units comprise an outboard terrane tectonically emplaced upon the ophiolitic unit. These upper units can be subdivided into two subunits: high-pressure/high-temperature (HPHT) and intermediate-pressure (IP) units. The HP-HT subunit contains eclogite- to granulite-facies paragneisses, ultramafic rocks, metagabbros and orthogneisses of continental and arc affinities. The age of the HP-HT metamorphism is a matter of debate, some authors favouring an Early Devonian age for this event (Ordoñez Casado et al., 2001) and others an Early Ordovician age (Kuijper, 1979; Fernández-Suárez et al., 2002b). The intermediate pressure unit (IP) is placed structurally on top of the HP-HT unit through a wide shear zone and consists of a sequence of low- to high-grade metasediments intruded by tholeiitic metagabbros 
VARISCAN GRANITES

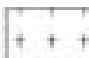

PARAUTOCHTHON

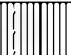

ALLOCHTHONOUS COMPLEXES: ORDENES COMPLEX
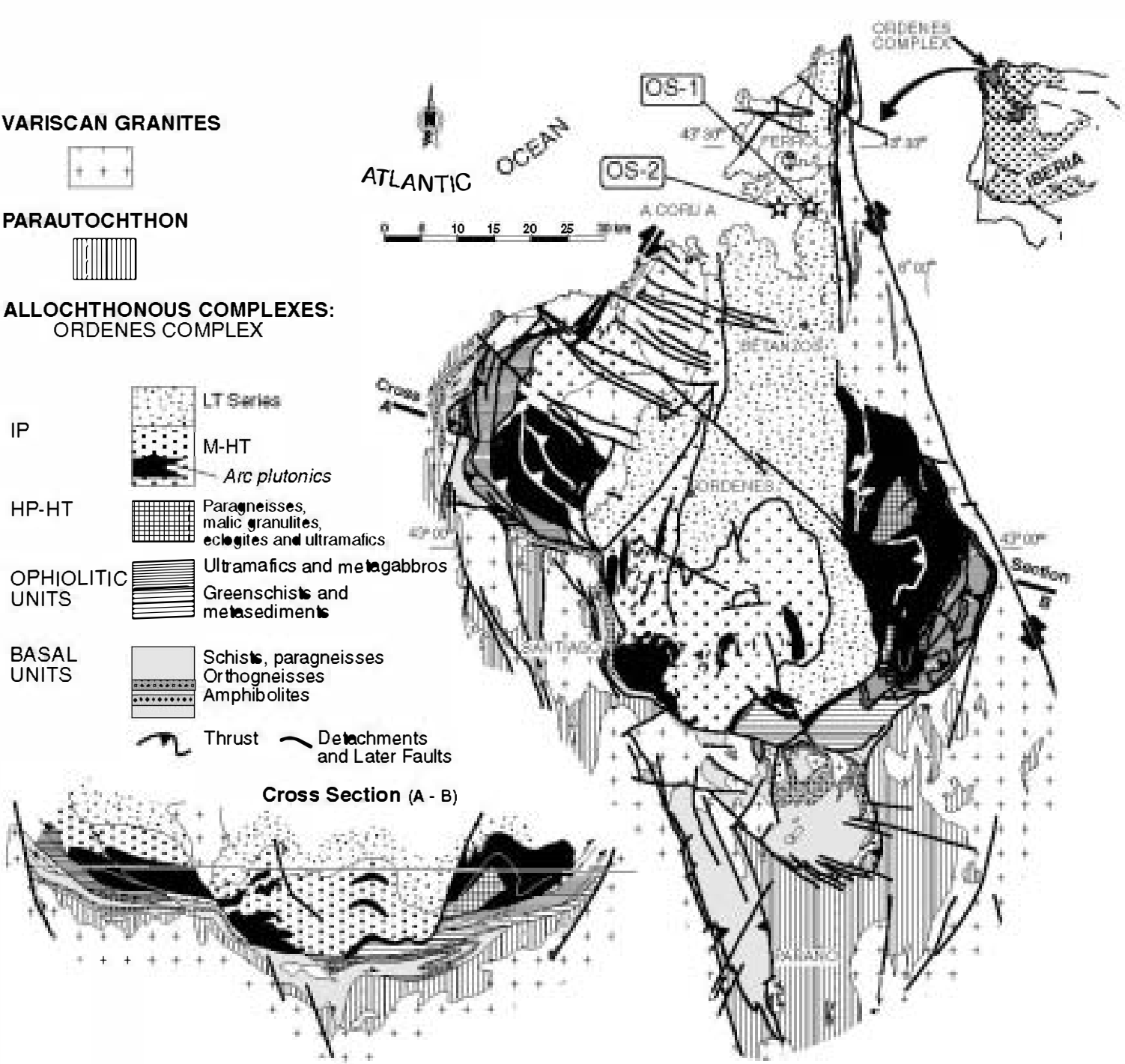

Fig. 1 Geological map and cross-section of the Ordenes Complex showing the main units and the location of greywacke samples. Illustration in the top right-hand corner shows autochthonous NW Iberia (Gondwanan margin) in a dotted pattern and the allochthonous complexes in grey.

and granodioritic to tonalitic orthogneisses at c. $500 \mathrm{Ma}$ (Abati et al., 1999). These intrusions have been interpreted as arc-related rocks (Abati, 2000).

The uppermost part of the IP unit of the outboard terrane (the Betanzos Unit) is made up of siliciclastic greenschist-facies rocks and is in tectonic contact with the underlying highertemperature metamorphic rocks. The lower part of the Betanzos Unit (Fig. 1) is a c. 1000-m-thick metasedimentary succesion consisting of black metapelites with alternations of quartzites. The upper part of the Betanzos unit consists of c. $1500 \mathrm{~m}$ of alternations of metagreywackes and slates with minor conglomerate levels. The whole unit is interpreted to represent a flyschoid succession deposited by a turbiditic system (Matte and Capdevilla, 1978; Gutiérrez Alonso et al., 2000) and has several characteristics of sediments deposited in amagmatic peri-arc basins. The abundance of plagioclase, volcanic quartz and fragments of igneous rocks, combined with the apparent lack of volcanic levels, suggest that this unit may represent a fore-arc basin fill (Diaz Garcia, 2000).

Two samples of coarse greywackes were collected in the upper part of the Betanzos Unit (OS-1 and OS-2, Fig. 1).

\section{Analytical techniques}

$\mathrm{U}-\mathrm{Pb}$ dating of individual zircon grains was performed using 213-nm laser ablation quadrupole ICP-MS at The Natural History Museum (London). The 213-nm laser system is a novel development for LA-ICP-MS $\mathrm{U}-\mathrm{Th}-\mathrm{Pb}$ isotope ratio determinations in zircons (Jeffries and Fernández-Suárez, 2001; Jeffries, 2001). Further details on the technique and analytical procedures are given elsewhere (Fernández-Suárez et al., 2000). Zircons separated by conventional techniques were set in synthetic resin mounts, polished and cleaned in a warm $\mathrm{HNO}_{3}$ ultrasonic bath. Catho- 
doluminiscence (CL) and back-scattered electron (BSE) imaging was performed and only zircons considered to be homogeneous on the basis of their CL/BSE images, or large homogeneous cores, were analysed. Analyses were performed using a 213-nm Nd:YAG laser ablation system (New Wave Research, USA) coupled to a quadrupole-based ICP-MS (PlasmaQuad 3, Thermo Elemental, UK) with an enhanced sensitivity (S-option) interface. To reduce the effects of interelement laser-induced fractionation, the zircons were ablated at the lowest power density required to couple to the sample (pulse energy $=0.15 \mathrm{~mJ}$ per pulse). During ablation, the sample was moved relative to the beam along raster or line patterns, appropriate to its size. For each determination, time-resolved signals were carefully studied to select stable, non-fractionated intervals, ensuring that inclusions, zonation and core-rim features were always excluded from age calculations.

\section{U-Pb results}

Results of 84 spot analyses of zircons are shown in Table 1 and in the concordia diagrams and histograms of Figs 2 and 3 . Although the analytical methodology and data reduction approach ensure that only isotopically homogeneous domains were considered for age calculations, analyses with discordancy higher than $30 \%$ were rejected. Furthermore, the ${ }^{207} \mathrm{~Pb} /$ ${ }^{2} \mathrm{~Pb}$ ages of all discordant analyses are consistent with $\mathrm{U}-\mathrm{Pb}$ concordant data points (Table 1), ensuring that age groups are not biased by the use of discordant analyses. U-Pb analytical results yielded the same zircon age populations in both samples and therefore data are presented together in Table 1 and Figs 2 and 3. This feature is consistent with the inferred equivalent stratigraphic position for both samples.

Samples OS-1 and OS-2 contain three main $\mathrm{U}-\mathrm{Pb}$ age groups (Table 1 , Figs 2 and 3). The younger group (c. $82 \%$ of the analyses) consists of zircons with ages ranging from c. $48 \mathrm{Ma}$ to c. $610 \mathrm{Ma}$ (Figs 2A and 3). The older groups consist of Palaeoproterozoic zircons in the age range c. $1950-2150 \mathrm{Ma}$ (c. $15 \%$ of the analyses) and 2450-2500 Ma
(Archean-Proterozoic limit) (c. 2-3\% of the analyses) (Figs $2 \mathrm{~B}$ and 3 ).

Within the first group, 37 analyses yielded Late Neoproterozoic ages (61 -540 Ma), 24 analyses yielded Cambrian ages (540-500 Ma) and eight analyses yielded Early Ordovician ages (500-48 $\mathrm{Ma}$ ).

\section{Discussion}

The data presented above provide a maximum depositional age for the Betanzos Unit at c. $48 \mathrm{Ma}$. There was no previous evidence for the age of the unit as neither palaeontological nor stratigraphic correlation criteria had constrained its stratigraphic age. This datum suggests that the deposition of the greywackes might have been coeval with the deposition of the Armorican Quartzite of the autochthonous terranes (Bonjour et al., 1988; FernándezSuárez et al., 2002a).

\section{Palaeogeographical links}

The main feature of the zircon age populations in the greywackes from the Betanzos Unit is the lack of Mesoproterozoic zircons (Fig. 3), at variance with detrital zircon age populations in Neoproterozoic and Palaeozoic sedimentary rocks of the Iberian autochthonous terrane, which contain abundant zircons in the age range 900-1200 Ma (FernándezSuárez et al., 2000, 2002a).

A second feature of interest is that the zircon age pattern found in the greywackes matches that of Neoproterozoic and Early Palaeozoic metasediments from the Ossa Morena Zone in SW Iberia (Gutiérrez Alonso et al., 2001), the north Armorican Domain (Miller et al., 2001) and zircon cores in gneisses from the Moldanubian and Saxothuringian zones of the Bohemian Massif (Tichomirowa et $\boldsymbol{a l}$., 2001, and references therein). This correspondence suggests a marked affinity of the Betanzos Unit with those domains of the European Variscides, which, in turn, are considered to have an affinity with the west African craton (Rocci et al., 1991, and references therein). Further to this, the age span of the youngest age group in the greywackes (48-61 Ma) corresponds to the main episodes of pre-Variscan magmatic activity recorded in the Ossa Morena Zone (Ordóñez Casado, 1998) and the north Armorican Massif (Chantraine et al., 2001, and references therein).

The presence of $c .2 \mathrm{Ga}$ zircons reflects the existence of a Palaeoproterozoic basement in the Variscan belt of western Europe (Icartian basement, e.g. Samson and D'Lemos, 1998) and in the west African craton (Eburnean basement, e.g. Rocci et al., 1991). The c. $2.5 \mathrm{Ga}$ zircons attest to the presence of an Archean basement component in the Variscan belt of western Europe (e.g. Guerrot et al., 1989).

The lack of Mesoproterozoic zircons suggests that the deposition of the greywackes took place in a realm with no access to detritus from either Laurentia or Baltica since recycling of cratonic areas of these continents would have yielded detrital material containing zircons in the age range c. 900-1600 Ma. The absence of Mesoproterozoic zircons is also an indication that recycling of pre-Neoproterozoic Amazonia or Neoproterozoic arcs developed on Amazonia were not involved at any stage in the evolution of the Betanzos Unit (cf. Fernández-Suárez et al., 2000) because Mesoproterozoic events are widespread in this cratonic area (e.g. Keppie et al., 2001, and references therein). The absence of Mesoproterozoic zircons further indicates that, at the time of deposition, the basin had no access to exposures of Precambrian-Cambrian autochthonous NW Iberia as recycling of these rocks would have also yielded zircons in the age range 900-1200 Ma (Fernández-Suárez et al., 2000).

The age spectra (c. 48-610,1900$2100,2400-2500 \mathrm{Ma}$ ) found in the greywackes match those of major events in the west African section of northern Gondwana, where no highgrade Mesoproterozoic events have been identified. Therefore, and in conjunction with the above arguments, we suggest that the periphery of the west African craton of northern Gondwana is the probable provenance of the upper units of the Allochthonous Complexes of NW Iberia.

Thoughts on possible tectonic settings Although further work is needed to refine the constraints on the pre-Variscan tectonic setting and evolution of the $\mathrm{AC}$, a few constraints can be drawn. 
Table 1 Laser Ablation ICP-MS U Pb analyses (concordant analyses in bold type)

\begin{tabular}{|c|c|c|c|c|c|c|c|}
\hline \multirow[b]{2}{*}{ Analysis no. } & \multicolumn{3}{|c|}{ Isotopic ratios ( $2 \sigma$ errors) } & \multicolumn{3}{|l|}{ Age (Ma) } & \multirow[b]{2}{*}{ Preferred age* $(\mathrm{Ma})$} \\
\hline & 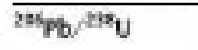 & ${ }^{200} \mathrm{~Pb} / 25 \mathrm{~S}_{\mathrm{U}}$ & ${ }^{851} \mathrm{~Pb} /{ }^{25 i} \mathrm{~Pb}$ & ${ }^{251} \mathrm{~Pb}{ }^{20} \mathrm{U}$ & ${ }^{201} \mathrm{~Pb} / 23 \mathrm{~V} \mathrm{~V}$ & ${ }^{207} \mathrm{~Pb} /{ }^{206} \mathrm{~Pb}$ & \\
\hline$\overline{a p 16 b 12}$ & $0.0901 \pm 0.0009$ & $0.7022 \pm 0.0090$ & $0.0565 \pm 0.0006$ & $556 \pm 11$ & $540 \pm 11$ & $472 \pm 50$ & $472 \pm 25$ \\
\hline ap16a09 & $0.0667 \pm 0.0008$ & $0.5217 \pm 0.0073$ & $0.0567 \pm 0.0004$ & $416 \pm 10$ & $426 \pm 10$ & $480 \pm 34$ & $480 \pm 17$ \\
\hline ap19a10 & $0.0780 \pm 0.0004$ & $0.6088 \pm 0.0031$ & $0.0567 \pm 0.0003$ & $484 \pm 5$ & $483 \pm 4$ & $478 \pm 24$ & $483 \pm 2$ \\
\hline ap16b10 & $0.0953 \pm 0.0011$ & $0.7469 \pm 0.0076$ & $0.0568 \pm 0.0007$ & $587 \pm 13$ & $566 \pm 9$ & $484 \pm 34$ & $484 \pm 17$ \\
\hline ap18a16 & $0.0791 \pm 0.0007$ & $0.6179 \pm 0.0060$ & $0.0566 \pm 0.0005$ & $491 \pm 9$ & $489 \pm 8$ & $476 \pm 34$ & $489 \pm 4$ \\
\hline ap20a12 & $0.0645 \pm 0.0006$ & $0.5072 \pm 0.0092$ & $0.0570 \pm 0.0010$ & $403 \pm 7$ & $417 \pm 12$ & $490 \pm 76$ & $493 \pm 38$ \\
\hline ap19b16 & $0.0582 \pm 0.0008$ & $0.4586 \pm 0.0061$ & $0.0571 \pm 0.0002$ & $365 \pm 10$ & $383 \pm 8$ & $496 \pm 16$ & $496 \pm 16$ \\
\hline ap16b16 & $0.0805 \pm 0.0004$ & $0.6295 \pm 0.0045$ & $0.0567 \pm 0.0003$ & $499 \pm 5$ & $496 \pm 6$ & $480 \pm 24$ & $498 \pm 10$ \\
\hline ap19a06 & $0.0801 \pm 0.0008$ & $0.6377 \pm 0.0057$ & $0.0578 \pm 0.0005$ & $496 \pm 10$ & $501 \pm 7$ & $520 \pm 38$ & $500 \pm 5$ \\
\hline ap19a15 & $0.0738 \pm 0.0005$ & $0.5824 \pm 0.0045$ & $0.0573 \pm 0.0003$ & $459 \pm 6$ & $466 \pm 6$ & $500 \pm 26$ & $500 \pm 26$ \\
\hline ap16b11 & $0.0925 \pm 0.0009$ & $0.7322 \pm 0.0078$ & $0.0574 \pm 0.0006$ & $570 \pm 10$ & $558 \pm 9$ & $506 \pm 42$ & $506 \pm 21$ \\
\hline ap19b13 & $0.0826 \pm 0.0014$ & $0.6527 \pm 0.0075$ & $0.0573 \pm 0.0008$ & $512 \pm 16$ & $510 \pm 9$ & $502 \pm 62$ & $510 \pm 10$ \\
\hline ap19a05 & $0.0565 \pm 0.0003$ & $0.4484 \pm 0.0021$ & $0.0576 \pm 0.0002$ & $354 \pm 3$ & $376 \pm 3$ & $512 \pm 12$ & $512 \pm 12$ \\
\hline ap17b13 & $0.0923 \pm 0.0009$ & $0.7348 \pm 0.0082$ & $0.0577 \pm 0.0004$ & $569 \pm 11$ & $559 \pm 10$ & $518 \pm 28$ & $518 \pm 14$ \\
\hline ap20a07 & $0.0837 \pm 0.0009$ & $0.6663 \pm 0.0096$ & $0.0577 \pm 0.0006$ & $518 \pm 11$ & $518 \pm 12$ & $518 \pm 44$ & $518 \pm 6$ \\
\hline ap20a06 & $0.0840 \pm 0.0016$ & $0.6695 \pm 0.0107$ & $0.0578 \pm 0.0010$ & $520 \pm 19$ & $520 \pm 13$ & $520 \pm 74$ & $520 \pm 6$ \\
\hline b06rim & $0.0780 \pm 0.0009$ & $0.6221 \pm 0.0086$ & $0.0578 \pm 0.0004$ & $484 \pm 10$ & $491 \pm 11$ & $522 \pm 28$ & $522 \pm 14$ \\
\hline ap17a06 & $0.0846 \pm 0.0004$ & $0.6753 \pm 0.0046$ & $0.0578 \pm 0.0004$ & $524 \pm 5$ & $524 \pm 6$ & $522 \pm 28$ & $524 \pm 3$ \\
\hline ap19b12 & $0.0718 \pm 0.0012$ & $0.5736 \pm 0.0106$ & $0.0579 \pm 0.0003$ & $447 \pm 15$ & $460 \pm 14$ & $524 \pm 20$ & $524 \pm 10$ \\
\hline ap17b14 & $0.0843 \pm 0.0012$ & $0.6771 \pm 0.0072$ & $0.0582 \pm 0.0003$ & $522 \pm 15$ & $525 \pm 9$ & $538 \pm 26$ & $528 \pm 4$ \\
\hline ap20a13 & $0.0793 \pm 0.0005$ & $0.6347 \pm 0.0055$ & $0.0580 \pm 0.0003$ & $492 \pm 6$ & $499 \pm 7$ & $528 \pm 24$ & $528 \pm 12$ \\
\hline ap17a16 & $0.0851 \pm 0.0011$ & $0.6875 \pm 0.0106$ & $0.0585 \pm 0.0008$ & $527 \pm 13$ & $531 \pm 13$ & $548 \pm 42$ & $529 \pm 6$ \\
\hline ap17b15 & $0.0725 \pm 0.0007$ & $0.5800 \pm 0.0072$ & $0.0580 \pm 0.0005$ & $451 \pm 8$ & $464 \pm 9$ & $530 \pm 40$ & $530 \pm 20$ \\
\hline ap16a05 & $0.0859 \pm 0.0006$ & $0.6689 \pm 0.0064$ & $0.0565 \pm 0.0005$ & $531 \pm 7$ & $520 \pm 8$ & $468 \pm 36$ & $531 \pm 7$ \\
\hline ap18b15 & $0.0861 \pm 0.0007$ & $0.6855 \pm 0.0064$ & $0.0577 \pm 0.0005$ & $532 \pm 8$ & $530 \pm 8$ & $518 \pm 38$ & $531 \pm 4$ \\
\hline ap20a10 & $0.0854 \pm 0.0008$ & $0.6878 \pm 0.0065$ & $0.0584 \pm 0.0005$ & $528 \pm 10$ & $531 \pm 8$ & $542 \pm 34$ & $531 \pm 5$ \\
\hline ap18a10 & $0.0730 \pm 0.0011$ & $0.5852 \pm 0.0126$ & $0.0581 \pm 0.0007$ & $454 \pm 13$ & $468 \pm 16$ & $532 \pm 54$ & $532 \pm 27$ \\
\hline ap19a07 & $0.0864 \pm 0.0007$ & $0.6927 \pm 0.0057$ & $0.0581 \pm 0.0005$ & $535 \pm 8$ & $534 \pm 7$ & $534 \pm 40$ & $534 \pm 3$ \\
\hline ap19a09 & $0.0569 \pm 0.0003$ & $0.4559 \pm 0.0029$ & $0.0581 \pm 0.0002$ & $357 \pm 4$ & $381 \pm 4$ & $534 \pm 16$ & $534 \pm 16$ \\
\hline ap18b09 & $0.0803 \pm 0.0011$ & $0.6441 \pm 0.0091$ & $0.0582 \pm 0.0006$ & $498 \pm 13$ & $505 \pm 11$ & $536 \pm 22$ & $536 \pm 22$ \\
\hline ap19b10 & $0.0780 \pm 0.0009$ & $0.6259 \pm 0.0074$ & $0.0582 \pm 0.0004$ & $484 \pm 11$ & $494 \pm 9$ & $536 \pm 32$ & $536 \pm 15$ \\
\hline ap20a14 & $0.0779 \pm 0.0014$ & $0.6256 \pm 0.0096$ & $0.0582 \pm 0.0006$ & $484 \pm 16$ & $493 \pm 12$ & $536 \pm 42$ & $536 \pm 21$ \\
\hline ap17a09 & $0.0876 \pm 0.0008$ & $0.7035 \pm 0.0061$ & $0.0582 \pm 0.0005$ & $541 \pm 10$ & $541 \pm 7$ & $534 \pm 34$ & $541 \pm 4$ \\
\hline ap20a11 & $0.0507 \pm 0.0006$ & $0.4085 \pm 0.0036$ & $0.0584 \pm 0.0004$ & $319 \pm 8$ & $348 \pm 5$ & $544 \pm 26$ & $544 \pm 12$ \\
\hline ap17a11 & $0.0775 \pm 0.0013$ & $0.6254 \pm 0.0078$ & $0.0585 \pm 0.0007$ & $481 \pm 16$ & $493 \pm 10$ & $546 \pm 48$ & $546 \pm 24$ \\
\hline ap17a14 & $0.0886 \pm 0.0010$ & $0.7099 \pm 0.0091$ & $0.0580 \pm 0.0009$ & $547 \pm 12$ & $545 \pm 11$ & $530 \pm 64$ & $546 \pm 5$ \\
\hline ap19a12 & $0.0737 \pm 0.0011$ & $0.5936 \pm 0.0100$ & $0.0585 \pm 0.0004$ & $458 \pm 13$ & $473 \pm 13$ & $546 \pm 32$ & $546 \pm 16$ \\
\hline ap16b05 & $0.0864 \pm 0.0006$ & $0.6969 \pm 0.0070$ & $0.0585 \pm 0.0004$ & $534 \pm 7$ & $537 \pm 8$ & $548 \pm 26$ & $548 \pm 13$ \\
\hline ap17b06 & $0.0885 \pm 0.0007$ & $0.7174 \pm 0.0072$ & $0.0588 \pm 0.0009$ & $547 \pm 8$ & $549 \pm 8$ & $558 \pm 66$ & $548 \pm 4$ \\
\hline ap16a10 & $0.0698 \pm 0.0006$ & $0.5641 \pm 0.0051$ & $0.0586 \pm 0.0002$ & $435 \pm 8$ & $454 \pm 7$ & $550 \pm 18$ & $550 \pm 9$ \\
\hline ap16b09 & $0.0902 \pm 0.0016$ & $0.7190 \pm 0.0099$ & $0.0578 \pm 0.0008$ & $557 \pm 18$ & $550 \pm 12$ & $520 \pm 62$ & $550 \pm 6$ \\
\hline ap17b05 & $0.0897 \pm 0.0007$ & $0.7164 \pm 0.0071$ & $0.0579 \pm 0.0005$ & $554 \pm 8$ & $549 \pm 8$ & $526 \pm 34$ & $551 \pm 5$ \\
\hline ap17a12 & $0.0766 \pm 0.0010$ & $0.6204 \pm 0.0097$ & $0.0587 \pm 0.0004$ & $476 \pm 12$ & $490 \pm 12$ & $554 \pm 28$ & $554 \pm 14$ \\
\hline ap17b07 & $0.0904 \pm 0.0009$ & $0.7244 \pm 0.0062$ & $0.0581 \pm 0.0005$ & $558 \pm 10$ & $553 \pm 7$ & $534 \pm 40$ & $554 \pm 4$ \\
\hline ap19a13 & $0.0720 \pm 0.0012$ & $0.5819 \pm 0.0095$ & $0.0587 \pm 0.0004$ & $448 \pm 14$ & $466 \pm 12$ & $554 \pm 26$ & $554 \pm 26$ \\
\hline ap18a07 & $0.0754 \pm 0.0006$ & $0.6115 \pm 0.0062$ & $0.0588 \pm 0.0005$ & $469 \pm 7$ & $484 \pm 8$ & $556 \pm 38$ & $556 \pm 19$ \\
\hline ap19b08 & $0.0830 \pm 0.0013$ & $0.6724 \pm 0.0078$ & $0.0587 \pm 0.0008$ & $514 \pm 15$ & $522 \pm 9$ & $556 \pm 56$ & $556 \pm 27$ \\
\hline ap16a12 & $0.1000 \pm 0.0007$ & $0.8111 \pm 0.0077$ & $0.0588 \pm 0.0004$ & $614 \pm 9$ & $603 \pm 9$ & $558 \pm 32$ & $558 \pm 16$ \\
\hline ap16а07 & $0.0774 \pm 0.0017$ & $0.6284 \pm 0.0134$ & $0.0588 \pm 0.0004$ & $481 \pm 21$ & $495 \pm 17$ & $560 \pm 28$ & $560 \pm 14$ \\
\hline ap17b10 & $0.0902 \pm 0.0010$ & $0.7410 \pm 0.0089$ & $0.0596 \pm 0.0011$ & $557 \pm 12$ & $563 \pm 10$ & $586 \pm 80$ & $560 \pm 5$ \\
\hline ap18a06 & $0.0817 \pm 0.0006$ & $0.6632 \pm 0.0062$ & $0.0588 \pm 0.0004$ & $506 \pm 8$ & $517 \pm 8$ & $560 \pm 28$ & $560 \pm 13$ \\
\hline ap16b14 & $0.0913 \pm 0.0010$ & $0.7364 \pm 0.0075$ & $0.0585 \pm 0.0007$ & $563 \pm 12$ & $560 \pm 9$ & $546 \pm 50$ & $561 \pm 5$ \\
\hline ap20a15 & $0.0909 \pm 0.0006$ & $0.7414 \pm 0.0045$ & $0.0591 \pm 0.0004$ & $561 \pm 7$ & $563 \pm 5$ & $572 \pm 30$ & $563 \pm 3$ \\
\hline ap17a07 & $0.0819 \pm 0.0009$ & $0.6665 \pm 0.0069$ & $0.0590 \pm 0.0004$ & $507 \pm 11$ & $519 \pm 8$ & $564 \pm 32$ & $564 \pm 16$ \\
\hline ap19a16 & $0.0936 \pm 0.0009$ & $0.7441 \pm 0.0063$ & $0.0577 \pm 0.0007$ & $577 \pm 10$ & $565 \pm 7$ & $516 \pm 52$ & $565 \pm 7$ \\
\hline ap18a05 & $0.0779 \pm 0.0007$ & $0.6350 \pm 0.0052$ & $0.0591 \pm 0.0003$ & $484 \pm 8$ & $499 \pm 6$ & $568 \pm 20$ & $568 \pm 10$ \\
\hline ap19b11 & $0.0811 \pm 0.0011$ & $0.6605 \pm 0.0092$ & $0.0591 \pm 0.0006$ & $503 \pm 13$ & $515 \pm 11$ & $568 \pm 46$ & $568 \pm 23$ \\
\hline ap18a12 & $0.0780 \pm 0.0009$ & $0.6367 \pm 0.0090$ & $0.0591 \pm 0.0006$ & $484 \pm 11$ & $500 \pm 11$ & $570 \pm 42$ & $570 \pm 20$ \\
\hline ap20a09 & $0.0926 \pm 0.0012$ & $0.7583 \pm 0.0143$ & $0.0594 \pm 0.0011$ & $571 \pm 15$ & $573 \pm 17$ & $578 \pm 82$ & $572 \pm 7$ \\
\hline
\end{tabular}


Table 1 (Continued)

\begin{tabular}{|c|c|c|c|c|c|c|c|}
\hline \multirow[b]{2}{*}{ Analysis no. } & \multicolumn{3}{|c|}{ Isotopic ratios ( $2 \sigma$ errors) } & \multicolumn{3}{|l|}{ Age (Ma) } & \multirow[b]{2}{*}{ Preferred age* $(\mathrm{Ma}$} \\
\hline & ${ }^{206} \mathrm{~Pb} /{ }^{238} \mathrm{U}$ & ${ }^{207} \mathrm{~Pb} /{ }^{235} \mathrm{U}$ & ${ }^{207} \mathrm{~Pb} /{ }^{206} \mathrm{~Pb}$ & ${ }^{206} \mathrm{~Pb} /{ }^{238} \mathrm{U}$ & ${ }^{207} \mathrm{~Pb} /{ }^{235} \mathrm{U}$ & ${ }^{207} \mathrm{~Pb} /{ }^{206} \mathrm{~Pb}$ & \\
\hline ap18a09 & $0.0616 \pm 0.0004$ & $0.5037 \pm 0.0040$ & $0.0592 \pm 0.0006$ & $386 \pm 5$ & $414 \pm 5$ & $574 \pm 42$ & $574 \pm 22$ \\
\hline ap17b12 & $0.0931 \pm 0.0011$ & $0.7647 \pm 0.0092$ & $0.0596 \pm 0.0008$ & $574 \pm 14$ & $577 \pm 11$ & $586 \pm 56$ & $576 \pm 5$ \\
\hline ap18a11 & $0.0684 \pm 0.0010$ & $0.5611 \pm 0.0085$ & $0.0594 \pm 0.0004$ & $426 \pm 12$ & $452 \pm 11$ & $582 \pm 26$ & $582 \pm 13$ \\
\hline ap19b07 & $0.0882 \pm 0.0007$ & $0.7228 \pm 0.0051$ & $0.0594 \pm 0.0004$ & $545 \pm 8$ & $552 \pm 6$ & $582 \pm 26$ & $582 \pm 26$ \\
\hline ap17a13 & $0.0889 \pm 0.0007$ & $0.7317 \pm 0.0064$ & $0.0596 \pm 0.0004$ & $549 \pm 8$ & $558 \pm 7$ & $588 \pm 28$ & $588 \pm 14$ \\
\hline ap18b08 & $0.0856 \pm 0.0009$ & $0.7057 \pm 0.0089$ & $0.0598 \pm 0.0006$ & $529 \pm 10$ & $542 \pm 11$ & $596 \pm 46$ & $596 \pm 23$ \\
\hline ap17b11 & $0.0896 \pm 0.0006$ & $0.7425 \pm 0.0079$ & $0.0601 \pm 0.0006$ & $553 \pm 7$ & $564 \pm 9$ & $604 \pm 42$ & $604 \pm 22$ \\
\hline ap18b05 & $0.0819 \pm 0.0009$ & $0.6788 \pm 0.0093$ & $0.0601 \pm 0.0009$ & $508 \pm 11$ & $526 \pm 11$ & $606 \pm 44$ & $606 \pm 31$ \\
\hline ap17a15 & $0.0992 \pm 0.0010$ & $0.8193 \pm 0.0071$ & $0.0599 \pm 0.0005$ & $609 \pm 12$ & $608 \pm 8$ & $598 \pm 34$ & $608 \pm 4$ \\
\hline ap18b14 & $0.0792 \pm 0.0014$ & $0.6579 \pm 0.0130$ & $0.0602 \pm 0.0005$ & $492 \pm 17$ & $513 \pm 16$ & $610 \pm 36$ & $610 \pm 18$ \\
\hline ap19b09 & $0.4155 \pm 0.0059$ & $6.9321 \pm 0.0624$ & $0.1210 \pm 0.0012$ & $2240 \pm 54$ & $2103 \pm 16$ & $1970 \pm 36$ & $1971 \pm 17$ \\
\hline ap16a13 & $0.4587 \pm 0.0041$ & $7.8892 \pm 0.0663$ & $0.1246 \pm 0.0009$ & $2434 \pm 36$ & $2218 \pm 15$ & $2022 \pm 26$ & $2022 \pm 26$ \\
\hline ap16b13 & $0.3550 \pm 0.0034$ & $6.1272 \pm 0.0466$ & $0.1252 \pm 0.0007$ & $1958 \pm 32$ & $1994 \pm 13$ & $2030 \pm 20$ & $2030 \pm 10$ \\
\hline ap16a06 & $0.3723 \pm 0.0030$ & $6.4972 \pm 0.0669$ & $0.1265 \pm 0.0009$ & $2040 \pm 28$ & $2046 \pm 18$ & $2048 \pm 26$ & $2046 \pm 10$ \\
\hline ap17a05 & $0.2875 \pm 0.0035$ & $5.0095 \pm 0.0616$ & $0.1262 \pm 0.0010$ & $1629 \pm 35$ & $1821 \pm 21$ & $2046 \pm 26$ & $2046 \pm 13$ \\
\hline ap19b05 & $0.3271 \pm 0.0035$ & $5.7224 \pm 0.0778$ & $0.1269 \pm 0.0007$ & $1824 \pm 34$ & $1935 \pm 24$ & $2054 \pm 20$ & $2054 \pm 20$ \\
\hline ap19a11 & $0.3419 \pm 0.0022$ & $5.9870 \pm 0.0383$ & $0.1270 \pm 0.0007$ & $1896 \pm 21$ & $1974 \pm 11$ & $2056 \pm 20$ & $2056 \pm 20$ \\
\hline ap17a08 & $0.3089 \pm 0.0037$ & $5.4265 \pm 0.1292$ & $0.1272 \pm 0.0016$ & $1735 \pm 36$ & $1889 \pm 41$ & $2060 \pm 44$ & $2060 \pm 22$ \\
\hline ap16b07 & $0.4079 \pm 0.0023$ & $7.2009 \pm 0.0475$ & $0.1280 \pm 0.0006$ & $2205 \pm 21$ & $2137 \pm 12$ & $2070 \pm 16$ & $2070 \pm 16$ \\
\hline ap19b14 & $0.3625 \pm 0.0025$ & $6.4196 \pm 0.0449$ & $0.1284 \pm 0.0004$ & $1994 \pm 23$ & $2035 \pm 12$ & $2076 \pm 12$ & $2076 \pm 12$ \\
\hline ap19a08 & $0.3514 \pm 0.0025$ & $6.3464 \pm 0.0324$ & $0.1310 \pm 0.0007$ & $1941 \pm 24$ & $2025 \pm 9$ & $2110 \pm 18$ & $2110 \pm 18$ \\
\hline ap18b12 & $0.3142 \pm 0.0048$ & $5.7188 \pm 0.0806$ & $0.1320 \pm 0.0003$ & $1761 \pm 47$ & $1934 \pm 24$ & $2124 \pm 8$ & $2124 \pm 8$ \\
\hline ap20a08 & $0.3573 \pm 0.0043$ & $6.6232 \pm 0.0854$ & $0.1343 \pm 0.0009$ & $1970 \pm 40$ & $2062 \pm 23$ & $2154 \pm 24$ & $2154 \pm 24$ \\
\hline ap18a13 & $0.3820 \pm 0.0029$ & $8.4559 \pm 0.0939$ & $0.1603 \pm 0.0010$ & $2086 \pm 27$ & $2281 \pm 20$ & $2458 \pm 20$ & $2458 \pm 20$ \\
\hline ap16a08 & $0.4751 \pm 0.0057$ & $10.5643 \pm 0.0750$ & $0.1612 \pm 0.0016$ & $2506 \pm 49$ & $2486 \pm 13$ & $2466 \pm 34$ & $2484 \pm 7$ \\
\hline ap16b06 & $0.3917 \pm 0.0045$ & $8.7933 \pm 0.1293$ & $0.1628 \pm 0.0010$ & $2131 \pm 41$ & $2317 \pm 27$ & $2484 \pm 20$ & $2484 \pm 20$ \\
\hline
\end{tabular}

*Age and error for concordant analyses are calculated according to Ludwig (1998). For discordant analyses the ${ }^{207} \mathrm{~Pb} /{ }^{206} \mathrm{~Pb}$ ages were used. Errors for discordant 'preferred ages' are those of upper intercept forced through $0 \pm 5 \mathrm{Ma}$.
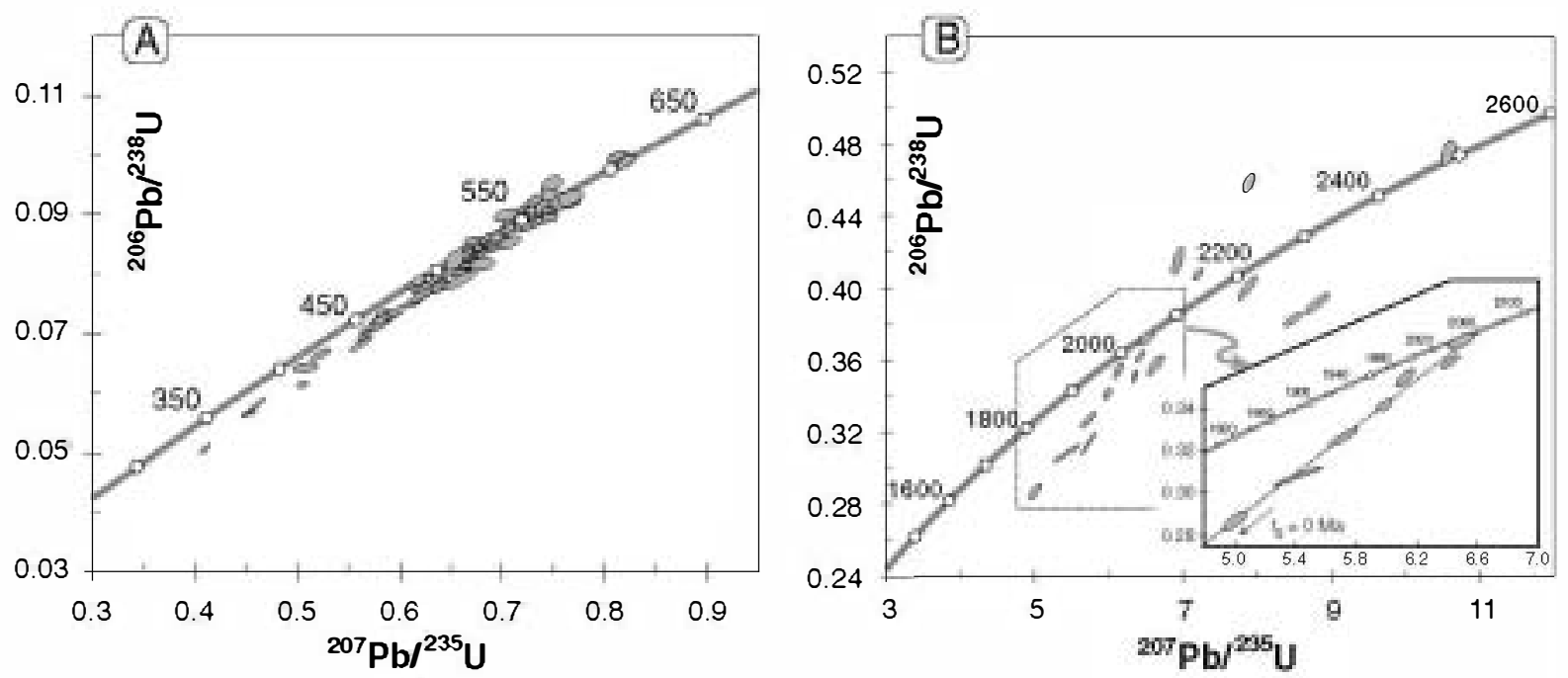

Fig. $2 \mathrm{U} \mathrm{Pb}$ concordia diagrams showing the results of LA ICP-MS dating of ircon (error ellipses represent $2 \sigma$ uncertainties). (A) Phanerozoic and Neoproterozoic zircons. (B) Palaeoproterozoic and Archaean zircons. Inset is shown to illustrate that a significant number of analyses have consistent ${ }^{207} \mathrm{~Pb} /{ }^{206} \mathrm{~Pb}$ ages around $2 \mathrm{Ga}$.

It is widely accepted that the Ordovician Armorican Quartzite was deposited in a platform bordering the African margin of Gondwana (e.g. Paris and Robardet, 1990) and it contains abundant Grenvillian zircons (Fernández-Suárez et al., 2002a). It has also been proposed that the source for those Grenvillian zircons is crustal fragments originated in the Amazo- nian margin of Gondwana and accreted to the African margin in the Early Palaeozoic by major strike slip faults (Fernández-Suárez et al., 2002a). In view of the arguments presented in the 

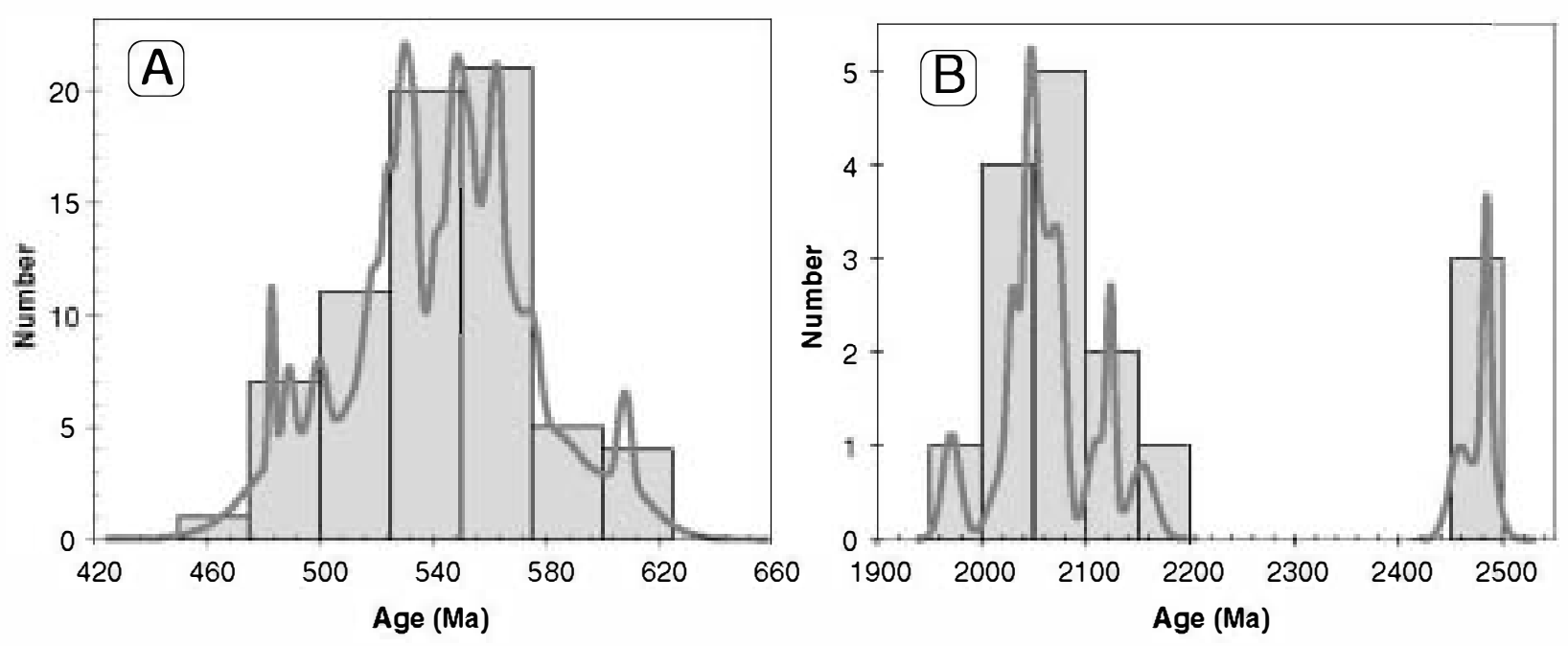

Fig. 3 Age histogram and cumulative probability plot. The plot has been constructed using the concordia ages and errors (Ludwig, 1998) for concordant analyses and the ${ }^{207} \mathrm{~Pb} /{ }^{206} \mathrm{~Pb}$ ages for discordant analyses. Errors for discordant analyses are derived from the upper intercept error of a discordia line forced through $\bullet \mathrm{Ma}$. A and B as in Fig. 2.

above section, we interpret this as an indication that the crustal element whose erosion produced the greywackes of the Betanzos Unit had been detached from the margin prior to the lateral accretion of Amazonia-derived blocks to the African margin. Therefore, at the time of deposition of the Armorican Quartzite on the Gondwanan platform there existed either a wide enough ocean separating the margin from the depositional locus of the greywackes or a barrier that impeded the access of detrital material from the continent. The latter possibility is compatible with a setting in which oceanic subduction and arc construction took place beneath the detached element. In such a case, the arc would have acted as a barrier for sediments shed by the continent and in this situation the basin separating the continent from the detached element need not be very wide. This scenario implies that the greywackes would have been deposited as fore-arc sediments, which is consistent with their sedimentological features (Díaz García, 2000).

In addition to the above, the U-Pb age spectra of detrital zircons in the greywackes of the Betanzos Unit are consistent with SHRIMP ages of zircon cores in high-grade metasediments of the underlying HP-HT units (Ordoñez Casado, 1998), suggesting that these units may represent variously metamorphosed and deformed sediments with the same source area and likely deposited in the same basin.
If this had been the case, and considering that the metasediments in the HP-HT units underwent high-grade and HP metamorphism at c. 500$490 \mathrm{Ma}$ (Fernández-Suárez et al., 2002b), then an environment where crustal subduction took place ought to be invoked to account for that contingency. In such a context, the greywackes could beconsidered to represent an overstepped sequence of an arc edifice under which the HP and IP units were buried and exhumed in a short time span (<10 Ma, Fernández-Suárez et al., 2002b). In this setting, the youngest zircon population of the greywackes might record dismantling of the c. $500 \mathrm{Ma}$ (Abati et al., 1999) arc rocks. However, available geological and geochronologic data do not allow further constraints to be placed on the features of this suggested setting.

\section{Synthesis}

The data and observations discussed above are consistent with the following hypothesis.

The upper units of the Allochthonous Complexes of NW Iberia represent a fragment of the African section of Neoproterozoic Gondwana that was detached from the margin in pre-Ordovician times as a result of extension following the main episode of Cadomian-Avalonian arc construction and terrane accretion (cf. Murphy et al., 2000). This detachment might have taken place in the context of the opening of the Rheic oceanic realm. The basin thus created separated the margin of Gondwana from a detached element made up of cratonic basement c. 2 Ga old (plus some Archean component) and Cadomian-Avalonian arc material, which constitute the main source for the greywackes of the upper units of the AC. In the Early Ordovician, this basin was wide enough for sediments from the margin to be unable to reach the greywacke basin. Alternatively, the greywackes could have been deposited as fore-arc sediments in a scenario of continentwards subduction and arc construction on the extended Gondwanan margin. In either case our contention is that the upper units of the AC may represent detached counterparts of Ossa Morena and the north Armorican Domain resulting from the Early Palaeozoic break-up of the Gondwanan margin that were eventually thrusted back upon it during the course of the Variscan collision.

\section{Acknowledgments}

J.F.S. wishes to acknøwledge a 'Cønt-

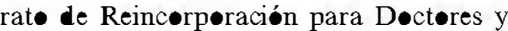
Tecnóløgos' from the Spanish MCYT. This study was funded through project PB97-0234-C 2 of the Spanish DGICYT. The Electron Microscopy and Mineral Analysis Division (NHM, Løndon) are kindly acknøwledged før technical and logistic support. Reviews by R. Strachan and J. B. Murphy are kindly appreciated. 


\section{References}

Abati, J., 2000. Petrología metamórfica y geocronología de la unidad culminante del Complejo de Ordenaes en la región de Carballo (Galicia, NIV del Macizo Ibérico). Unpublished doctoral Dissertation, UCM, Madrid.

Abati, J., Dunning, G.R., Arenas, R., Díaz García, F., Gønzález Cuadra, P. and Martínez Catalán, J.R., 1999. Early Ordøvician $\bullet$ regenic event in Galicia (NW Spain): Evidence from U-Pb ages in the uppernost unit of the Ordenes Complex. Earth Planet. Sci. Lett., 165, 213-228.

Arenas, R., Rubiø Pascual, F.J., Diaz García, F. and Martínez Catalán, J.R., 1995. High-pressure micrø-inclusions and development of an inverted metamorphic gradient in the Santiage Schists (Ordenes Complex, NW Iberian Massif, Spain): evidence of subduction and syncøllisiønal decompression. J. Metam. Geol., 13, 141-164.

Bønjøur, J.L., Peucat, J.J., Chauvel, J.J., Paris, F. and Cornichet, J., 1988. U-Pb zircon dating of the Early Paleøzoic (Arenigian) trans gression in western Brittany (France): a new constraint for the Løwer Paleøzic time-scale. Chem. Geol., 72, 329-336.

Chantraine, J., Eqal, E., Thiéblemønt, D., Le G॰ff, E., Guerrøt, C., Ballevre, M. and Guennøc, P., 2001. The Cadomian active margin (North Ann七rican Massif, France): a segment of the North Atlantic Panafrican belt. Tectonophysics, 331, 1-18

Díaz García, F., 2000. Lith $\bullet$ stratigraphy and Structure of the A Coruña Unit. Uppernıst Units $\bullet$ the Variscan Belt, NW Spain. In: Variscan-Appalachian dynamics: the building of the Upper Paleozoic Basement. Basement Tectonics 15, A Coruña, Spain, Program Abstracts, $8,92-94$

Díaz García, F., Arenas, R., Martínez Catalán, J.R., Gønzález del Táną̧, J and Dunning, G.R., 1999. Tectonic ev॰lution $\bullet$ f the Careon $\bullet p h i \bullet l i t e$ (Northwest Spain): a remnant of oceanic Lith $\bullet$ sphere in the Variscan belt. J. Geol., 107, 587-605.

Fernández-Suárez, J., Cørfu, F., Arenas, R., Marcœs, A., DíaZ. García, F., Martínez Catalán, J.R., Abati, J. and Fernández, F.J., 2002b. U-Pb evidence

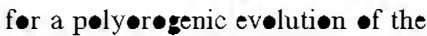
HP-HT units of the NW Iberian Massif Contrib. Miner. Petrol., in press.

Fernández-Suárez, J., Gutiérrez Aløns•, G., C•x, R. and Jenner, G.A., 2002a. Assembly of the Armorica micrøplate: a strike-slip terrane delivery? Evidence frøm U-Pb ages of detrital zircøns. $J$. Geol., in press.

Fernández-Suárez, J., Gutiérrez Aløns•, G., Jenner, G.A. and Tubrett, M.N.,
2000. New ideas on the PrøterøzicEarly Pale $\bullet \bullet i c$ evelution of NW Iberia. Insights fr $\bullet$ U-Pb detrital zircon ages. Precambrian Res., 102, 185-206.

Guerrøt, C., Peucat, J.J. and Capdevila, R., 1989. Archean proteliths within Early Pr ter zoic ranulitic crust of the west Eurøpean Hercynian belt: pøssible relics of the west African craton. Geology, 17, 241-244.

Gutierrez Alıns•, G., Barba, P. and DíaZ. García, F., 2000. Sedimentelegy and structure of the low rade uppermest unit of the Ordenes Complex at the cœastal section. Superpesition of structural trends and processes. In: VariscanAppalachian dynamics: the building of the Upper Paleozoic Basement. Basement Tectonics 15, A Coruña, Spain, Program Abstracts, 8, 234-236.

Gutiérrez-Aløns•, G., Fernández-Suárez, J., Jeffries, T.E., Jenner, G.A. and Cøx, R., 2001. Tøwards a deconstruction of the western European CadomianAvalonian belt: detrital zircens tell their tale. IGCP Project no. 453 Meeting: Collisional Orogens, Sion, Abstracts and Programme, 65-67. University of Lausanne, Switzerland.

Jeffries, T., 2001. 213 nm Laser Ablation ICP-MS- rev•lution or gimmick? Invited Lecture at European Winter Conference on Plasma Spectrochemistry. Hafjell, Norway: Conference Abstracts, p. 79. The Norwegian Chemical Seciety.

Jeffries, T and Fernández-Suárez, J., 2001. Zircon dating-213nm versus $266 \mathrm{Nd}$ : YAG lasers. Application Note. New Wave Research, Merchantek Prøucts, California

Keppie, J.D., Destal, J., Ortega-Gutiérrez, F. and López, R., 2001. A Grenvillian arc $\bullet$ the margin of Amaz»nia: evidence from the southern Oaxacan complex, søuthern Mexice. Precambrian Res., 112, 165-181.

Kuijper, R.P., 1979. U-Pb systematics and the petrogenetic evolution of in fracrustal rocks in the Paleozoic basement of western Galicia (NIV Spain). Unpublished decteral Dissertation. ZWO Laberatery Is॰t. Ge•l., Amsterdam.

Ludwig, K.R., 1998. On the treatment of concordant uranium-lead ages. Geochim. Cosmochim. Acta, 62, 665-676.

Martínez Catalán, J.R., Arenas, R., Díaz. García, F. and Abati, J., 1997. The Variscan accretionary complex of NW Iberia: invelved terranes and succession -f tectøn thernal events. Geology, 25, 1103-1106.

Martínez Catalán, J.R., Arenas, R., Díaz García, F. and Abati, J., 1999. Alløchth-nøus units in the Variscan belt of NW Iberia. Basement Tectonics, 13, 65-84.

Martínez Catalán, J.R., Arenas, R., Díaz García, F., Rubi Pascual, F.J., Abati, J and Marquínez, J., 1996. Variscan exhumation of a subducted Pale $\bullet \bullet i c$ continental margin: the basal units of the Ordenes Complex, Galicia, Spain. Tectonics, 15, 106-121.

Matte, $\mathrm{Ph}, 2001$. The Variscan collage and -røgeny (480-290 Ma) and the tectonic definition of the Amørica micrøplate: a review. Terra Nova, 13, 122-128.

Matte, $\mathrm{Ph}$ and Capdevilla, R., 1978. Tectonique en grand plis couches et plissements superpeses d'age hercynien dans la serie de Ordenes-Betanzes (Galice -ccidentale). Cuad. Sem. Est. Geol. Cerámica Sargadelos, 27, 195-201.

Miller, B.V., Samsøn, S.D. and D'Lemes, R.S., 2001. U-Pb geochrønelegical constraints on the timing of plutenism, volcanism, and sedimentation, Jersey, Channel Islands, UK. J. Geol. Soc., 158 243-252.

Murphy, J.B., Strachan, R.A., Nance, R.D., Parker, K.D. and Føwler, M.B., 2000. Pr t•-Avalønia: a 1.2-1.0 Ga tecton thermal event and constraints for the evølution of Rødinia. Geology, 28 1071-1074.

Ordóñez Casade, B., 1998. Geochronological studies of the pre-Mesozoic basement of the Iberian Massit: the Ossa Morena zone and the Alloch thonous complexes within the Central Iberian Zone. Unpublished dectøral Dissertatiøn, ETH, Zurich.

Ordónez Casad•, B., Gebauer, D., Schäffer, H.J., Ibarguichi, J.I.G. and Peucat, J.J., 2001. A single Devenian subduction event for the HP/HT metamørphism of the Cab• Ortegal complex within the Iberian Massif. Tectonophysics, 332, 359-385.

Paris, F. and Røbardet, M., 1990. Early

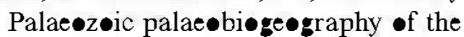
Variscan regions. Tectonophysics, 177, 193-213.

Røcci, G., Brøner, G. and Deschamps, M., 1991. Crystalline basement of the west African craton. In: The West African Orogens and Circum-Atlantic Correlatives ( $\mathrm{R}$. D. Dallmeyer and J. P. Lecorche, eds), pp. 31-61. Springer Verlag, Berlin.

Samsøn, S.D. and D'Lem»s, R.S., 1998. $\mathrm{U}-\mathrm{Pb}$ geochroneløy and Sm-Nd is -

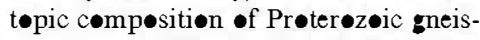
ses, Channel Islands, UK. J. Geol. Soc., 155, 609-618.

Tichømirøwa, M., Berger, H.J., K•ch, E.A., Belyatski, B.V., G̈̈tze, J., Kempe, U., Nasdala, L. and Schaltegger, U., 2001. Zircon ages of high-grade gneisses in the Eastern Erzgebirge (Central Eurøpean Variscides) - cønstraints •n -rigin of the rocks and Precambrian to Ordovician magmatic events in the Variscan foldbelt. Lithos, 56, 303-332. 